\title{
A pseudo anapole based electromagnetic cloaking scheme using Split Ring Resonators
}

\section{Sarin VP ( $\nabla$ sarincrema@gmail.com )}

University of Calicut

\section{Vinesh PV \\ University of Calicut}

\section{Manoj M}

Cochin University of Science and Technology

\section{Mohanan P}

Cochin University of Science and Technology

\section{Vasudevan $\mathrm{K}$}

Cochin University of Science and Technology

\section{Research Article}

Keywords: cylindrical electromagnetic cloaking scheme, pseudo-anapole, Split-ring resonators, toroidal moments

Posted Date: October 11th, 2021

DOI: https://doi.org/10.21203/rs.3.rs-750483/v2

License: (c) (i) This work is licensed under a Creative Commons Attribution 4.0 International License. Read Full License 


\title{
A pseudo anapole based electromagnetic cloaking scheme using Split Ring Resonators
}

\author{
Sarin V.P ${ }^{1}$, Vinesh P.V ${ }^{1}$,Manoj $\mathrm{M}^{2}$, P.Mohanan ${ }^{2}$, and Vasudevan Kesavath ${ }^{2}$ \\ ${ }^{1}$ Department of Electronics, Government College Chittur, Palakkad, Kerala-678104, India \\ ${ }^{2}$ Centre for Research in Electromagnetics and Antennas, Cochin University of Science and \\ Technology, Cochin-22, Kerala, India
}

Abstract: This paper proposes the first experimental demonstration of a pseudo-anapole-based cylindrical electromagnetic cloaking scheme. The pseudo-anapole state is excited by arranging split-ring resonators around a cylindrical metallic target.We used the multipole scattering theory to identify the actual reason behind the cloaking operation. Pseudo anapole condition is characterized by the scattering minima from toroidal and electric dipole moments, and hence the metallic target is undetectable from backscattering measurements. The results are verified using full-wave simulation software and subsequently validated with backscattering measurements inside an anechoic chamber.

\section{Introduction}

The experimental realization of the electromagnetic invisibility cloaking scheme has attracted much research interest recently. The term cloaking refers to the significant reduction of scattering from reference metallic or dielectric targets. Mathematically, it refers to the effective reduction of the Scattering Cross Section (SCS) of the target, including the incident direction and all directions prone to scattering. The undetectability of the target finds applications in the area of sensors, Radars etc. It is a well-known fact that when the electric and magnetic dipoles induced on the sub-wavelength dielectric sphere oscillate out-of-phase, then the SCS of the composite will be significantly reduced [1]. This conclusion is known as Kerker's paradox, and many studies are available on this particular research topic [2]. The problem with this scheme is the unavailability of natural magnetic materials used to create magnetic dipole moments at microwave frequencies. J.B Pendry et al. showed that the Split-Ring Resonators (SRR) made of concentric metallic split rings could achieve artificial magnetism at microwave frequencies [3]. This invention is treated as a paradigm shift in electromagnetics because we could control the amplitude and phase of electric and magnetic moments individually to achieve unprecedented control over electromagnetic scattering. 
J.B Proposed the practical demonstration of a cloaking scheme to make a copper cylinder invisible using single split-ring resonator metamaterials [4]. The combination of the target and the metamaterial creates an electromagnetic environment similar to space. Scattering cancellation-based cloaking is also implemented using plasmonic covers over dielectric objects [5]. The dielectric target's scattered field is canceled in plasmonic cloaking due to the anti-phase scattering from the negative permittivity outer layer. The mantle cloaking technique is used to cloak both the dielectric and metallic targets, and its basic idea relies on tuningthe surface reactance of the FSS layer to cancel far-field scattering [6]. Recently, Fano resonance-based scattering cancellation techniques have attracted significant research interest [7]. Fano resonance arises due to the destructive interference between the bright, resonant mode of the continuum and the dark asymmetric resonant mode.It is worth mentioning that the magnetic dipole transitions are approximated to be $10^{5}$ times weaker than the corresponding electric dipole transition, and hence the optical frequency regime is said as a world of pure electric dipoles [8]. So the creation of Fano resonance is an excellent choice to excite strong magnetic dipole excitation in the visible regime[9]. The intense excitation of magnetic dipoles is used for electromagnetic cloaking in the microwave regime [10].

Recently, a higher-order multipole, known as the toroidal moment, has attracted significant research interest due to itsunique scattering characteristics. Toroidal dipoles are created due to the poloidal currents flowing on the composite [11-12], and their scattering behaviour is similar to that of an electric dipole [13]. In conventional metamaterials structures, the excitation of these moments will be significantly weak. When the size of the unit cell approaches the wavelength of operation, a significant excitation of toroidal moments is observed. The excitation of toroidal moments adds an additional degree of freedom to control electromagnetic scattering [14]. Since the scattering characteristics of the electric dipole and the toroidal dipole are similar, an out-ofphase excitation of these moments results in invisibility cloaking. The combination of toroidal and electric dipole moments is termed as an anapole. An anapole excitation is characterized by the destructive interference between the electric and toroidal moments subject to the condition $P=-\mathrm{ikT}$, where $\mathrm{P}$ is the electric dipole moment, $\mathrm{T}$ is the toroidal moment and $\mathrm{k}$ is the wavenumber. Recently a wide variety of works has been focused on the anapole excitation in metamaterials [15-16]. There is a close relationship between the V-th Devaney-Wolf theorem 
regarding a nonradiating current distribution and the anapole mode [17]. The equivalent current distribution $J_{e q}$ corresponding to the anapole can be written as

$$
J_{e q}=\nabla X \nabla X T-i k P=0
$$

Basharin et. al. proposed another solution to achieve invisibility by obeying the condition $\mathrm{P}=0$ and $\mathrm{T}=0$. Besides the anapole condition $(\mathrm{P}=-\mathrm{ikT})$, the minimal value of $\mathrm{P}$ and $\mathrm{T}$ also achieves the non-radiating condition [18]. They called this condition as the pseudo-anapole mode. In pseudoanapole excitation, radiation from the fundamental electric dipole moment is significantly suppressed compared to the higher-order multipoles. The suppressed electric and toroidal moments significantly reduce the far-field scattering from the system resulting in invisibility.

This paper proposes the first experimental demonstration of the pseudo anapole based electromagnetic invisibility cloaking. The pseudo anapole condition is achieved by arranging the SRR particles around a cylindrical metallic target. The arrangement of SRR around the target excites strong electric dipole resonance, and the scattering caused by them destructively interferes with that from the non-resonant electric dipole moment of the target to make the target invisible. We used the multipole scattering theory to validate the reason behind the scattering reduction. Experiments are performed inside an anechoic using theAnritsuMS2027CNetwork analyzer to validate the full-wave simulation results.

\section{Geometrical Description}

For experimental and simulation studies, we constructed three prototypes. The first one, designated as case 1, consists of a cylindrical metallic target surrounded by only two SRR strips, as shown in fig. 1(a). The azimuth displacement between the two strips is $180^{\circ}$. Eight SRRcells are arranged vertically in a strip, and the assembly consists of 16 SRR inclusions. The length of the target cylinder is $160 \mathrm{~mm}$, and its radius is $5 \mathrm{~mm}$. The dimensions of the SRR used are shown in the inset of fig. 1(a). In the second design (case2), we used four SRR strips arranged around the target, as shown in fig. 1(b). The total number of SRR in this design is 32 . In the third design (case 3), the number of SRR strips is increased to 8, as shown in fig. 1(c). The SRR strips are printed on an epoxy substrate of dielectric constant 4.4 and height $1.6 \mathrm{~mm}$ using the standard photo-lithographic procedures. The thickness of the implanted Copper metalization is $35 \mu \mathrm{m}$. 
The dimensions of the SRR used are $\mathrm{r}=6.7 \mathrm{~mm}, \mathrm{~d}=2 \mathrm{~mm}, \mathrm{~s}=0.8 \mathrm{~mm}, \mathrm{w}=1 \mathrm{~mm}, \mathrm{~h}=1.6 \mathrm{~mm}$, and $\mathrm{p} 1=\mathrm{p} 2=20 \mathrm{~mm}$.

(a)

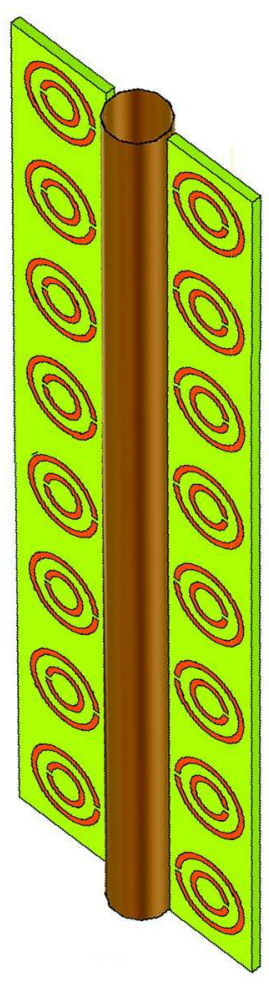

(b)

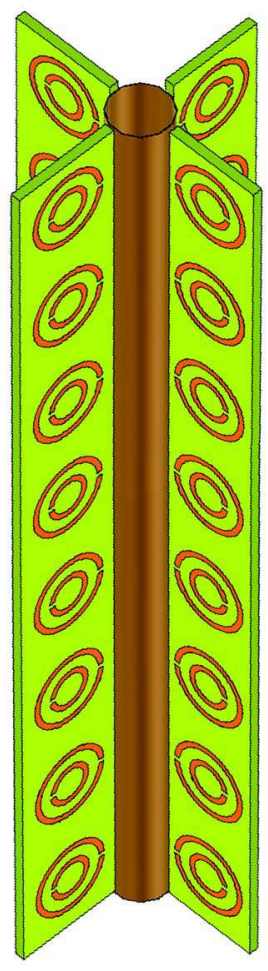

(c)

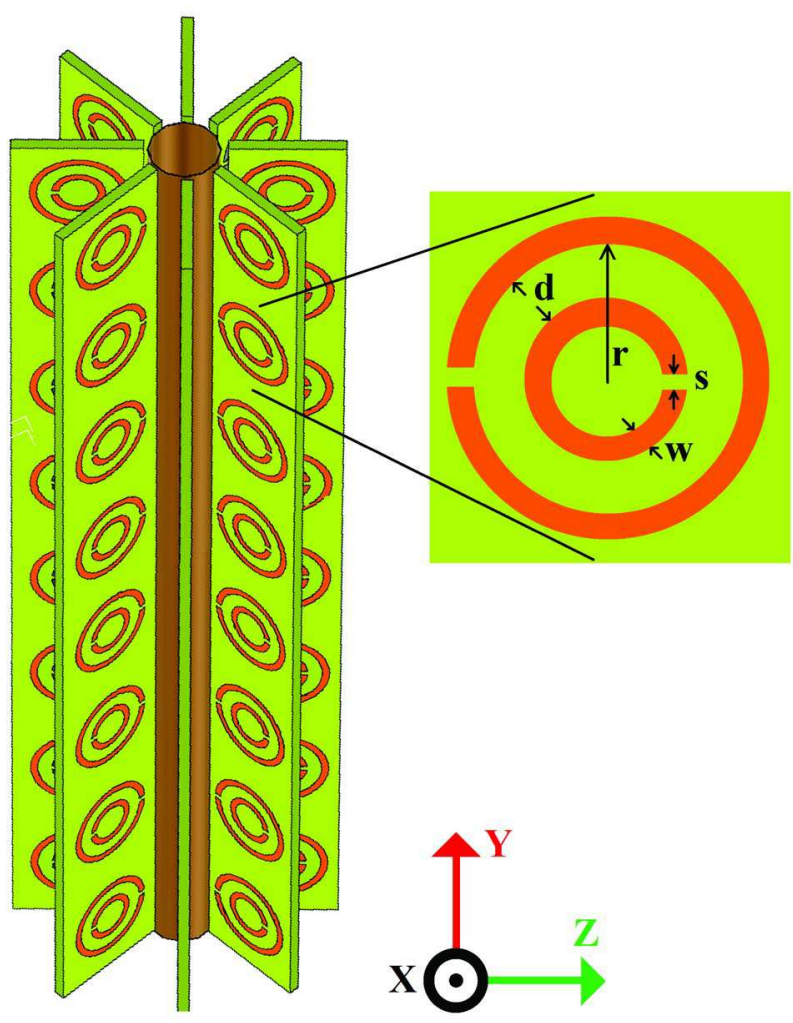

Fig.1 Geometry of the cloaking scheme a) two-layer cloak (case 1), b) Four-layer cloak (case 2), and c) Eight layer cloak (case 3) with the description of SRR dimensions in the inset.

\section{Simulation Results}

We performed full-wave simulation studies on the three prototypes using CST Microwave Studio. The three structures are analyzed independently in the simulation by exciting the complete structure with a plane wave. The polarization of the plane wave is oriented along the Y-axis. The computed Scattering Cross Section (SCS) of the three structures are illustrated in fig. 2. The scattering dip for case 1 is observed at $1.95 \mathrm{GHz}$, and it shows comparable scattering characteristics to the uncloaked metallic target.Case 2 reduces the SCS of the target significantly, and a well-defined resonant scattering reduction is observed. Case 2 shows a maximum scattering reduction at $1.9 \mathrm{GHz}$. Due to the increase in the mutual coupling between 
the SRR elements, a significant redshift is observed for case 3. The resonance is observed to be at $1.6 \mathrm{GHz}$ for this design, and since this design shows the maximum scattering reduction, it is taken as the optimum design.

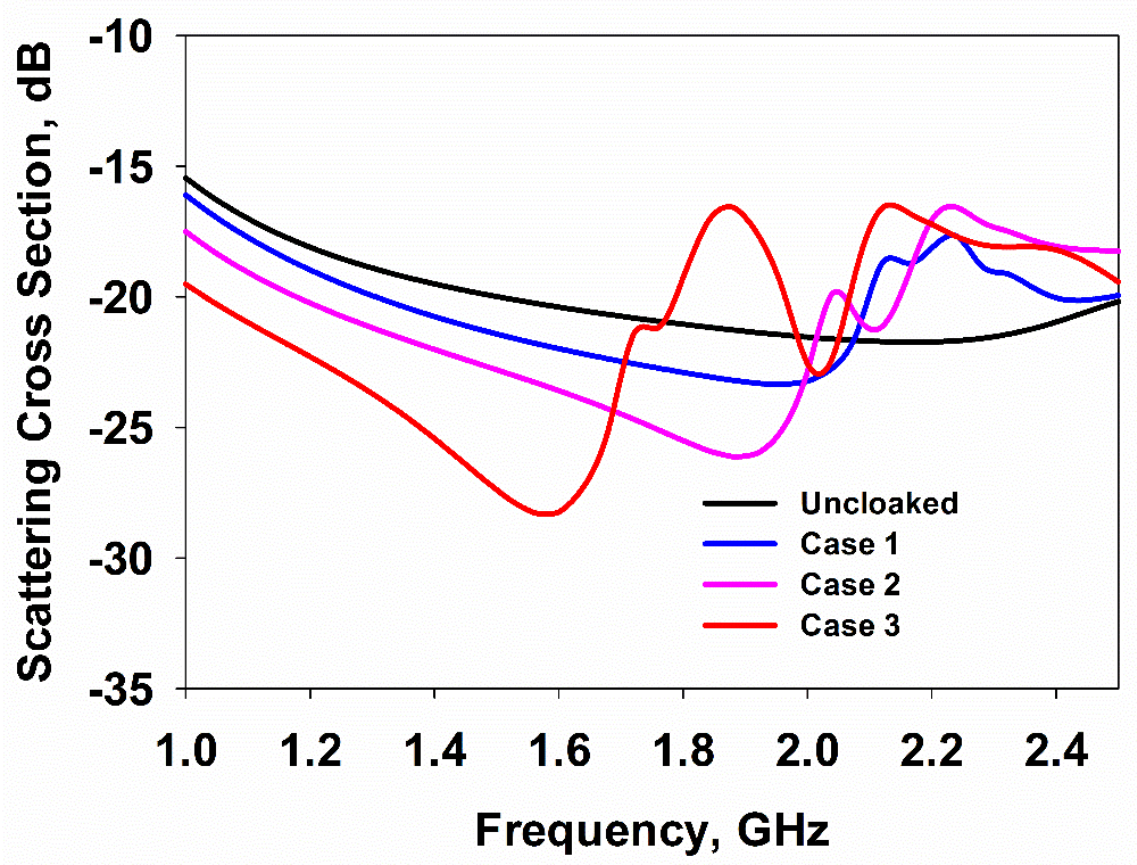

Fig.2 Scattering Cross Sections of the three prototypes

To understand the cloaking operation, the field distributions are studied for the cloaked and uncloaked target. Fig. 3 shows the simulated field distributions on the uncloaked target under consideration. One could observe from fig. 3(a) that the uncloaked cylinder blocks 
(a)
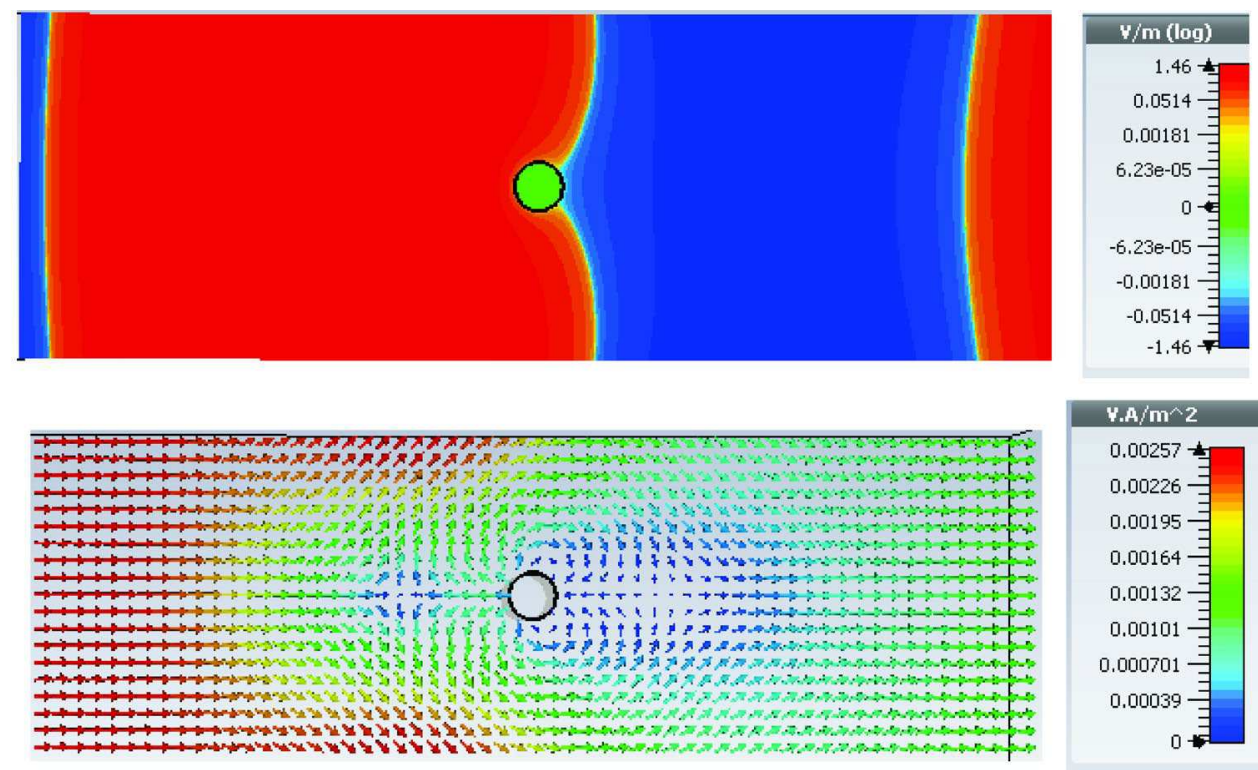

Fig. 3 Results of numerical simulation for the uncloaked target at $1.6 \mathrm{GHz}$ a) Electric field distribution from the YZ plane and b) Pointing vector distribution from the XZ plane

The significant perturbation of electromagnetic power is also evident from the Pointing power distribution shown in fig. 3(b). 
(a)

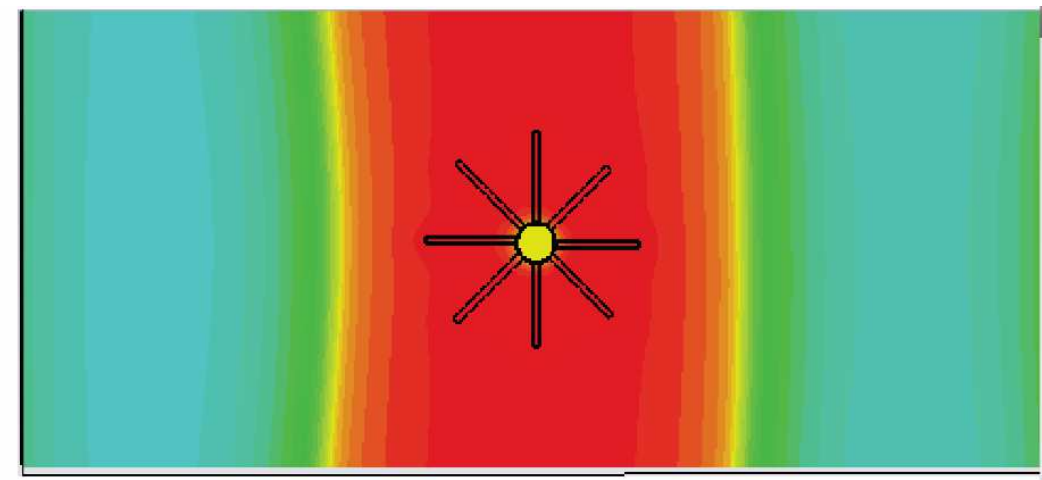

(b)

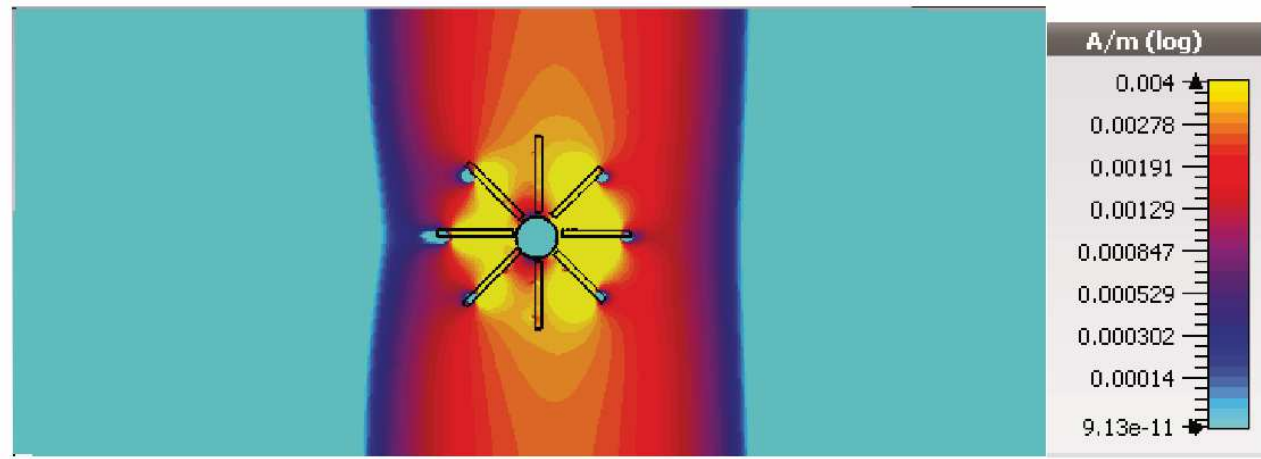

(c)

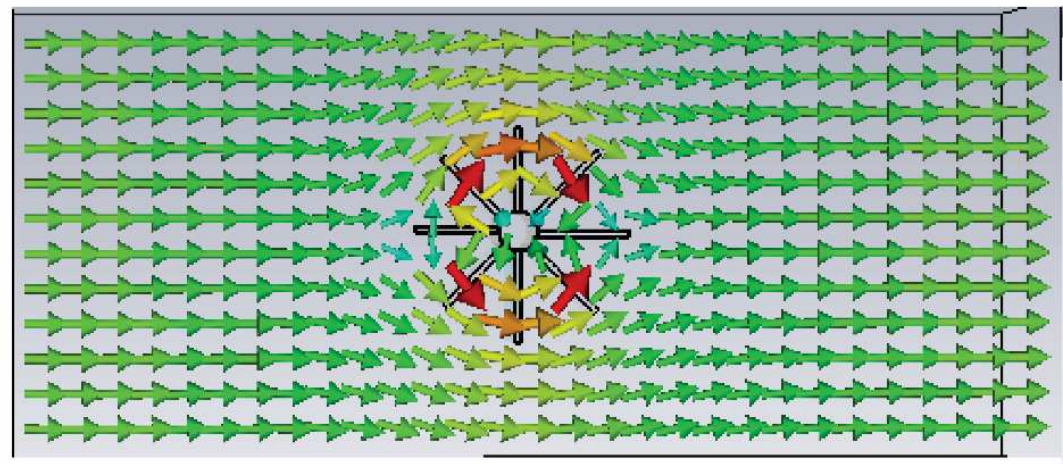

\section{$V . A / m^{\wedge} 2(\log )$}

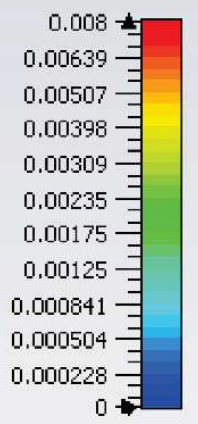

Fig. 4 Computed fields of the cloaked target at $1.6 \mathrm{GHz}$ a) Electric field distribution, b) magnetic field distribution, and c) Pointing vector distribution

The computed field distributions for the cloaked target (case 3) are shown in fig. 4. A smooth flow of electric and magnetic fields is observed in the computational domain, as shown in fig. 4(a) and (b) respectively. This means that the cloaking structure significantly reduces scattering from the metallic target under consideration. The Pointing vector distribution, shown in fig. 4(c) illustrates a smooth flow of electromagnetic power, and the shadow observed in the uncloaked target is absent in the cloaked scenario. The SRR cloak cover transfers smoothly or 
bends the incident electromagnetic wave into the output face, and no scattering is observed around the cloaking layer.

The scattering characteristics of the cloaked and uncloaked targets are also verified by computing the 3D scattering patterns. Fig. 5(a) shows the scattering pattern of the uncloaked target. The uncloaked target scatters electromagnetic power equally in the azimuth plane, and two principal nulls are observed along theelevation plane. The non-resonant electric dipole excitation is responsible for this scattering mechanism, and hence the target is detectable from far-field scattering measurements. Fig. 5(b) shows the scattering pattern of the cloaked target (Case 3). A significant reduction in scattered power is observed at the far-field around the target.

(a)

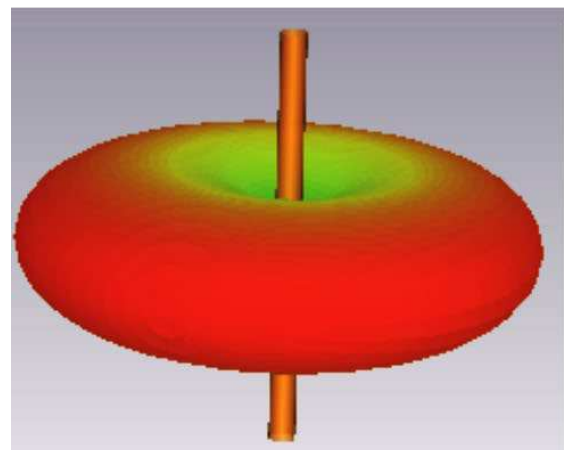

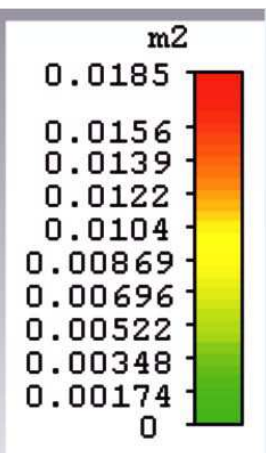

(b)

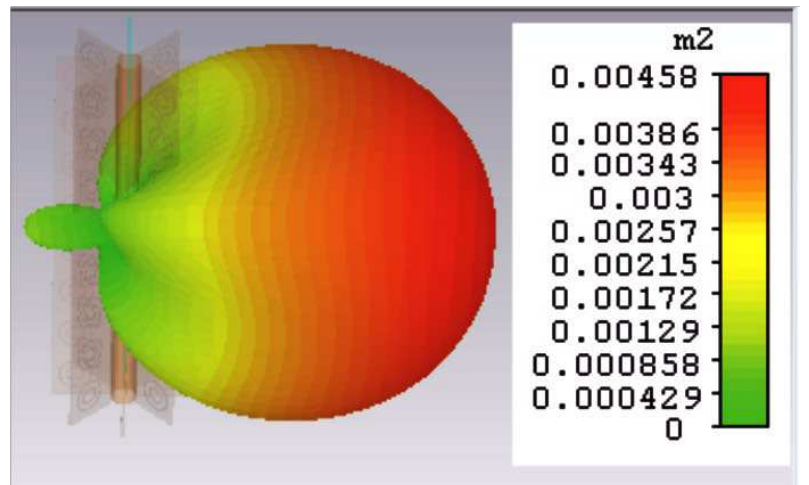

Fig. 5 Scattering patterns of the cloaked and uncloaked targets a) uncloaked target and b) cloaked target

\section{Experimental Results}

The scattering characteristics of the three prototypes are verified using backscattering measurementsusinga network analyzer. To measure backscattering, the uncloaked target is placed at the center of a turntable assembly. Two ultra-wideband antennas are utilized for the measurement. One antenna is configured in the transmission mode and the second one in the reception mode. A THRU calibration is performed by connecting the two RF cables,and Frequency Gated by Time (FGT) calibrationis applied to notchout unwanted noise signals received from other directions. The resultant received power is taken as the reference, and 
finally, the cloaked target is placed instead of the bare metallic cylinder target. The backscattered power thus received is recorded using the interface computer. For bistatic measurements, the receiving horn antenna is rotated along the azimuth angle using the turntable assembly, and the corresponding backscattered powersare recorded for the three fabricated prototypes. Since the eight-layer structure (case 3) showsa better reduction in backscattered power, it is taken as the optimum design. Fig. 5 shows the backscattered power from the fabricated designs. Fig. 5(a) shows the backscattered power from the two-layer (Case1) design. It is evidentthat the design shows poor backscattering characteristics compared to the other two designs. For this case, the backscattered power is comparable in magnitude for most of the azimuth angle as that of the bare metallic target. It is interesting to note that as the number of SRR layers around the target increases, the backscattered power is decreased, as shown in fig. 5(b) and (c). Case 2 shows better backscattering characteristics compared to case 1. A significant reduction in backscattering is observed for the eight-layer design (Case 3)compared to the other two designs. A maximum backscattering reduction of the order of $-16 \mathrm{~dB}$ is observed for this design. 
(a)

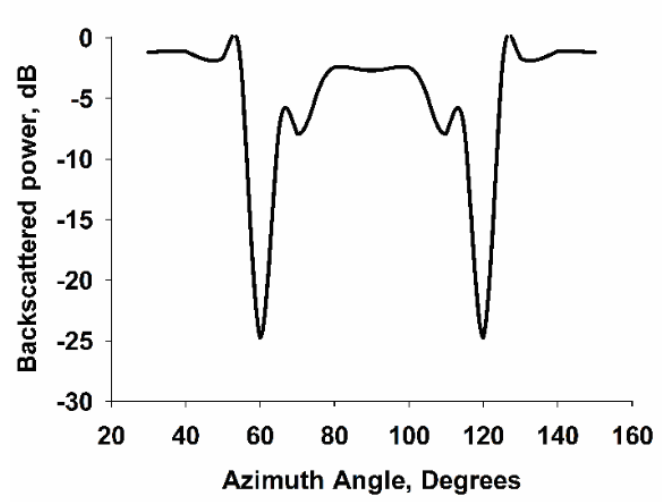

(b)

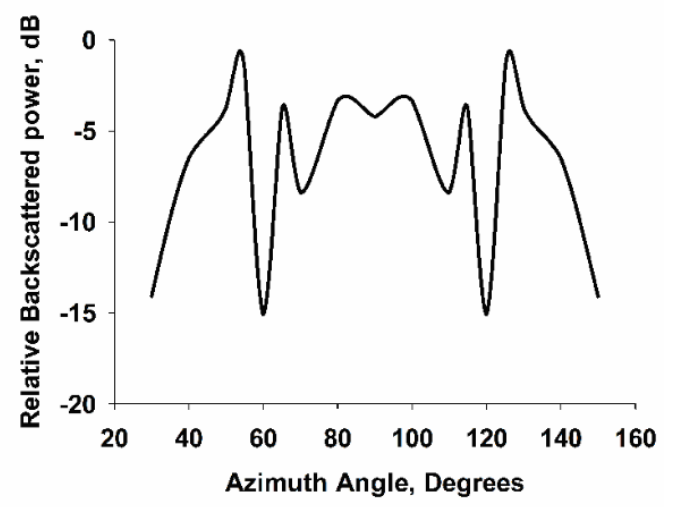

(c)

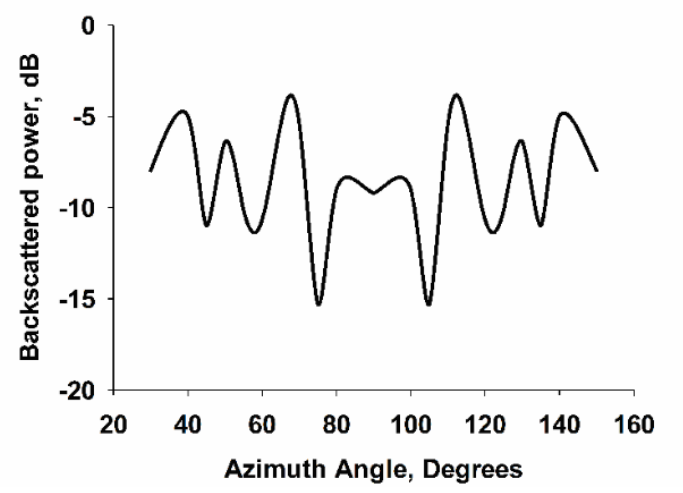

Fig. 5Backscattered power from the fabricated samples a) Case 1, b) case 2, and c) Case 3

We have also performed the monostatic scattering measurements to compare the scattering performance of the three fabricated prototypes. In monostatic measurements, the power received from the uncloaked target located at the turntable assembly center is taken as the reference after performing the FGT calibration. The cloaked target is rotated along the azimuth plane while two UWB antennas are kept fixed.The received power thus obtained is plotted in fig. 6 for the three configurations under study. It is evident from the graph that the eight-layer configuration (case 3) gives a better backscattering reduction for all azimuth rotations of the target. It is also noted that the two-layer (case 1) and four-layer (case 2) structures also exhibit scattering reduction compared to a bare metallic target. 


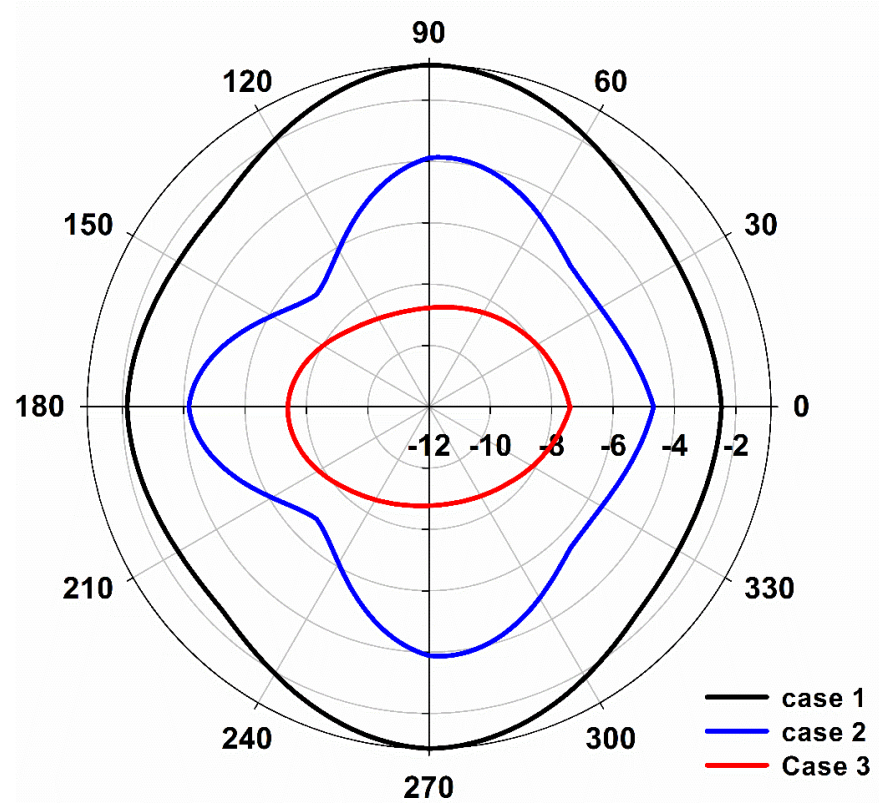

Fig. 6MonostaticScattering characteristics of the fabricated configurations

\section{Discussions}

To clarify the reason behind this peculiar scattering behavior, multipole scattering theory has been utilized to retrieve the structure's resonant mechanism [13].The multipolar decomposition provides an in-depth description of the scattering properties of the composite due to the induced charge-current distributions. Scattered power from the induced multipoles could be calculated by integrating spatially distributed current distributions of the unit cell. The multipole amplitudes can be calculated as

$$
\begin{gathered}
P=\frac{1}{i \omega} \int J d^{3} r \\
\left.M=\frac{1}{2 c} \int \overrightarrow{(r} X J\right) d^{3} r \\
\left.P=\frac{1}{10 c} \int[\overrightarrow{(r} \cdot J)-2 r^{2} J\right] d^{3} r
\end{gathered}
$$

where $\mathrm{P}$ is the electric dipole moment, $\mathrm{M}$ is the magnetic dipole moment, $\mathrm{T}$ is the toroidal moment, $\mathrm{c}$ is the velocity of light in vacuum, $\underset{r}{\overrightarrow{\text { is }}}$ the displacement vector from the origin, $\omega$ is the angular frequency, and $\mathrm{J}$ is the surface current density retrieved from simulations. The total power radiated from different multipole moments can be formulated as 


$$
I=\frac{2 \omega^{4}}{3 \mathrm{c}^{3}}|P|^{2}+\frac{2 \omega^{4}}{3 \mathrm{c}^{3}}|M|^{2}+\frac{2 \omega^{6}}{3 \mathrm{c}^{5}}|T|^{2}+\ldots \ldots .
$$

(a)

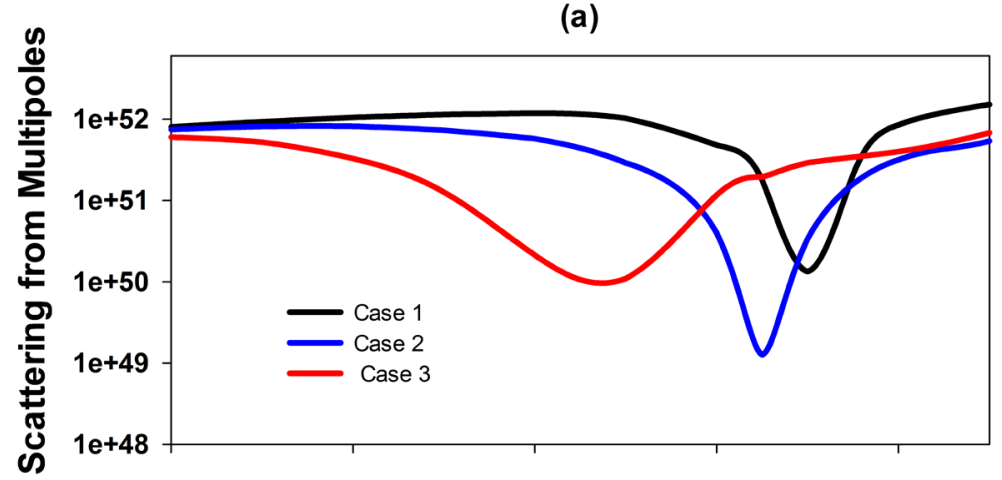

(b)

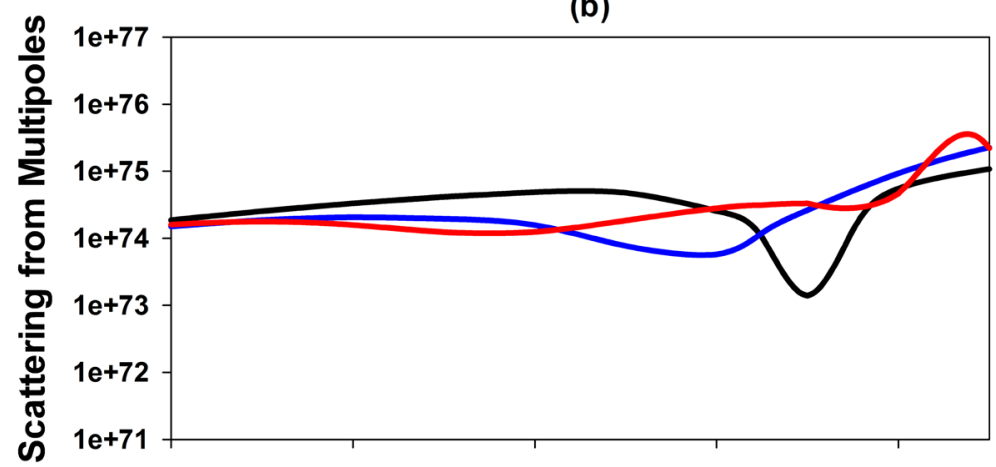

(c)

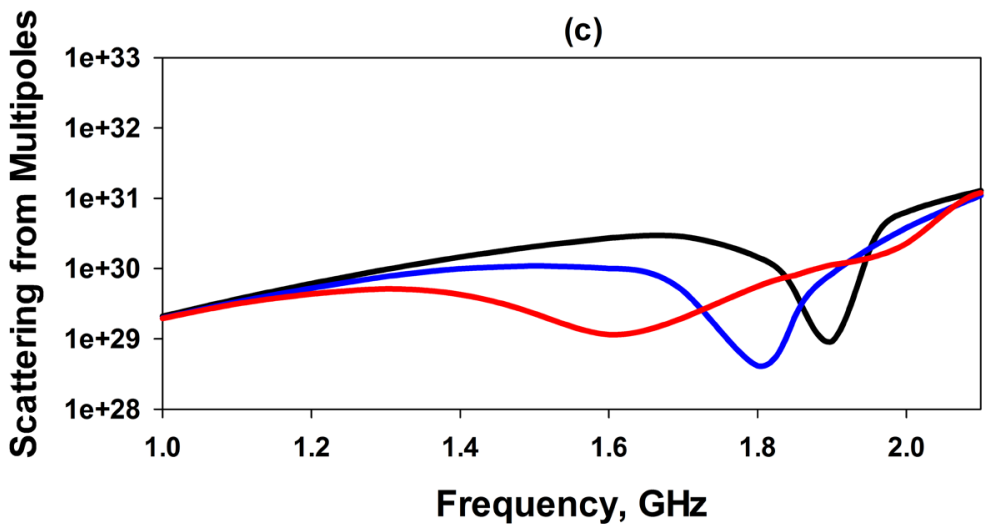

Fig. 7Scatteringcontribution from a) electric dipole moment $\left(P_{y}\right)$, b) Magnetic dipole moment $\left(M_{x}\right)$ and $\left.c\right)$ Toroidal dipole moment $\left(T_{y}\right)$ 
significantly,resulting in electromagnetic invisibility. It is to be noted that the perfect pseudoanapole condition, $\mathrm{P}=\mathrm{ikT}=0$, cannot be observed in this scenario. As pointed out by Basharin [], the electric and toroidal scattering dip is sufficient to achieve a non-radiating equivalent current distribution.

\section{Conclusions}

This paper has studied and presented the first experimental verification of a pseudo-anapolebased cylindrical electromagnetic cloaking scheme using a Split-Ring Resonator array in the microwave regime. The excitation of the pseudo-anapole state significantly reduces the scattering from the electric and toroidal dipole moments at the far-field resulting in a reduction in the Scattering Cross Section of the target. These results are verified using the multiple scattering theory and experimentally verified in the microwave regime inside an anechoic chamber.

\section{Methods}

The simulation studies of the proposed designs are performed in the frequency domain using the commercially available CST Microwave Studio. The complete structure is illuminated with a plane wave with polarization parallel to the axis of the metallic target to study the scattering characteristics. Inorder to increase the accuracy of computations, adaptive mesh refinement is used. Field monitors are used to extract the electrical currents excited on the composite, and mathematical computations are performed on these retrieved current distributions using the GNU Octave software to calculate the power radiated from different multipoles.

\section{REFERENCES}

1. Kerker, M., Wang, D.S. \& Giles, L. Electromagnetic scattering by magneticspheres. J. Opt. Soc. Am. 73, 765-767 (1983).

2. Milligan, T.A. Modern Antenna Design, IEEE, John Wiley \& Sons, 2005.

3. J. B. Pendry, A. J. Holden, D. J. Robbins, and W. J. Stewart, Magnetism from Conductors and EnhancedNonlinear Phenomena, IEEE Trans. Microw. Theory and tech., 47, 11, 2075-2084 (1999) 
4. David Schurig, Jack J Mock, B.J Justice, Steven A Cummer, John B. Pendry, Anthony F Starr, David R Smith, Metamaterial electromagnetic cloak at microwave frequencies, Science, 314, 5801 (2006)

5. Andrea Alù \& Nader Engheta 2005. Achieving transparency with plasmonic and metamaterial coatings. Physical Review E. 72(016623):1-9

6. A. Alù, Mantle cloak: invisibility induced by a surface. Phys. Rev. B 80, 245115 (2009)

7. K.B. Samusev, M.V. Rybin, A.K. Samusev, M.F. Limonov, Invisibility of a finite dielectric cylinder under Fano resonance conditions, Phys. Sol. State,57, 1991-1996 (2015)

8. Tim H. Taminiau, Sinan Karaveli, Niek F. van Hulst \& Rashid Zia. Quantifying the Magnetic Nature of Light Emission. Nature Communications. 3, 979, 1-6(2012)

9. FarbodShafiei, Francesco Monticone,Khai Q. Le, Xing-Xiang Liu, Thomas Hartsfield, Andrea Alu, and Xiaoqin Li, A subwavelength plasmonic metamolecule exhibiting magnetic-based optical Fano resonance, Nat. Nano Tech., 8, 95-99 (2013)

10. Barbara Cappello, Anar K. Ospanova, LadislauMatekovits, Alexey A.Basharin, Mantle cloaking due to ideal magnetic dipole scattering, Nat. Scientific Reports, 10, 2413 (2020)

11. Zel'dovich IB The relation between decay asymmetry and dipole moment of elementary particles. Journal of Experimental and Theoretical Physics 6, 1184. (1958)

12. Kaelberer T, Fedotov VA, Papasimakis N, Tsai DP and Zheludev NI Toroidal dipolar response in a metamaterial. Science (New York, N.Y.) 330, 1510-1512(2010)

13. Afanasiev GN and Stepanovsky YP The electromagnetic field of elementary timedependent toroidal sources. Journal of Physics A: Mathematical and General 28, 4565. (1995)

14. Terekhov PD, Baryshnikova KV, Shalin AS, Karabchevsky A and Evlyukhin $\mathrm{AB}$,Toroidal dipole associated resonant forward scattering of light by Silicon nanoparticles. Progress In Electromagnetics Research Symposium - Spring (PIERS). (2017)

15. Miroshnichenko AE, Evlyukhin AB, Yu YF, Bakker RM, ChipoulineA,Kuznetsov AI, Luk'yanchuk B, Chichkov BN and Kivshar YS,Nonradiating anapole modes in dielectric nanoparticles. Nature Communications 6, 8069. (2015) 
16. Nemkov NA, Stenishchev IV and Basharin AA Nontrivial nonradiating all-dielectric anapole. Nat. Sc. Rep. 7, 1064. (2017)

17. G Labate, AK Ospanova, NA Nemkov, AA Basharin, L Matekovits, Nonradiating anapole condition derived from Devaney-Wolf theorem and excited in a brokensymmetry dielectric particle, Optics Express 28 (7), 10294-10307 (2020)

18. Maria V Cojocari, Anar K Ospanova, Vladimir I Chichkov, Miguel Navarro-Cia, Andrei Gorodetsky, Alexey A Basharin., Pseudo-anapole regime in terahertz metasurfaces, Phys. Rev. B 104, 075408 (2021)

\section{Acknowledgements}

The authors acknowledge the research funding received from the Science and Engineering Research Board (SERB), Department of Science and Technology for the major research project ECR/2017/002204.

\section{Author Contributions}

Sarin V.P made a substantial contribution to the conception, design, fabrication, simulation, and measurement of the research work. Vinesh P.V performed the simulation and measurements of the cloaking structures. Manoj M, Mohanan $\mathrm{P}$, and Vasudevan $\mathrm{K}$ participated in the simulation and computational studies of the paper.

\section{Additional Information}

Competing financial interests: The authors declare no competing financial interests. 